\title{
What is the Incidence of Kidney Stones after Chemotherapy in Patients with Lymphoproliferative or Myeloproliferative Disorders?
}

Hossein S. Mirheydar'1, Pooya Banapour², Rustin Massoudi², Kerrin L. Palazzi ${ }^{1}$, Ramzi Jabaji2, Erin G. Reid $^{3}$, Frederick E. Millard ${ }^{3}$, Christopher J. Kane ${ }^{1,2,4}$, Roger L. Sur ${ }^{1,2,4,5}$

${ }^{1}$ Department of Urology, UC San Diego Health System, San Diego CA, USA; ${ }^{2} U C$ San Diego School of Medicine, La Jolla, CA, USA; ${ }^{3}$ Department of Medicine, Division of Hematology/Oncology, UC San Diego Health System, San Diego, CA, USA; ${ }^{4} V A$ San Diego Healthcare System, San Diego, CA, USA, ${ }^{5}$ Uniformed Services University of the Health Sciences, Bethesda, MD, USA

\section{ABSTRACT}

Introduction: This study describes the incidence and risk factors of de novo nephrolithiasis among patients with lymphoproliferative or myeloproliferative diseases who have undergone chemotherapy.

Materials and Methods: From 2001 to 2011, patients with lymphoproliferative or myeloproliferative disorders treated with chemotherapy were retrospectively identified. The incidence of image proven nephrolithiasis after chemotherapy was determined. Demographic and clinical variables were recorded. Patients with a history of nephrolithiasis prior to chemotherapy were excluded. The primary outcome was incidence of nephrolithiasis, and secondary outcomes were risk factors predictive of de novo stone. Comparative statistics were used to compare demographic and disease specific variables for patients who developed de novo stones versus those who did not.

Results: A total of 1,316 patients were identified and the incidence of de novo nephrolithiasis was 5.5\% (72/1316; symptomatic stones 1.8\% 24/1316). Among patients with nephrolithiasis, $72.2 \%$ had lymphoproliferative disorders, $27.8 \%$ had myeloproliferative disorders, and 25\% utilized allopurinol. The median urinary $\mathrm{pH}$ was 5.5 , and the mean serum uric acid, calcium, potassium and phosphorus levels were 7.5, 9.6, 4.3, and $3.8 \mathrm{mg} / \mathrm{dL}$, respectively. In univariate analysis, mean uric acid ( $\mathrm{p}=0.013)$, calcium $(\mathrm{p}<0.001)$ ), and potassium $(p=0.039)$ levels were higher in stone formers. Diabetes mellitus $(p<0.001)$, hypertension $(p=0.003)$, and hyperlipidemia $(p<0.001)$ were more common in stone formers. In multivariate analysis, diabetes mellitus, hyperuricemia, and hypercalcemia predicted stone.

Conclusions: We report the incidence of de novo nephrolithiasis in patients who have undergone chemotherapy. Diabetes mellitus, hyperuricemia, and hypercalcemia are patient-specific risk factors that increase the odds of developing an upper tract stone following chemotherapy.

\section{ARTICLE INFO}

\section{Key words:}

Urolithiasis; Chemotherapy, Adjuvant; Calculi; Kidney Calculi; Ureteral Calculi

Int Braz J Urol. 2014; 40: 772-80

Submitted for publication:

March 05, 2014

Accepted after revision:

June 23, 2014

\section{INTRODUCTION}

Tumor lysis syndrome (TLS) is an oncologic emergency observed among patients with hematologic malignancies associated with significant morbidity/mortality if untreated. TLS occurs when tumor cells release their contents into the bloodstream, either spontaneou- 
sly or after chemotherapy, leading to hyperuricemia, hyperkalemia, hyperphosphatemia, and hypocalcemia (1). Such metabolic sequelae are known to cause renal insufficiency, cardiac arrhythmias, seizures, and death.

The increased rate of cell turnover associated with proliferative disorders and chemotherapy induced cell turnover may result in an increased rate of urate nephropathy and nephrolithiasis (2). This results from the rapid release of intracellular macromolecules that are metabolized to phosphorous and uric acid at a pace that may exceed the patient's clearance capacity. The hyperphosphatemia may result in precipitation of calcium phosphate crystals and lead to increased nephrolithiasis (3). Lastly, hyperuricemia may lead to increased nephrolithiasis via intrarenal precipitation (4).

Lymphoproliferative disorders are a set of diseases characterized by the abnormal proliferation of lymphocytes into a monoclonal population, and include a wide spectrum of diagnostic entities (e.g., follicular lymphoma, chronic lymphocytic leukemia (CLL), acute lymphoblastic leukemia (ALL), hairy cell leukemia, lymphomas, multiple myeloma, and Waldenstrom's macroglobulinemia). Myeloproliferative disorders are bone marrow stem cell disorders and include chronic myeloid leukemia, polycythemia vera, essential thrombocythemia, and idiopathic myelofibrosis, which all have potential to transform into acute leukemias.

While it is well established that lymphoproliferative/myeloproliferative disorders and tumor lysis can result in hyperuricosuria and hyperuricemia, there is limited literature describing the incidence of nephrolithiasis in this select population. There are case reports documenting nephrolithiasis among patients receiving chemotherapy for these disorders (5-7), and a single study examined a larger cohort (5). Moreover, risk factors for nephrolithiasis in patients undergoing chemotherapy have been identified in the pediatric literature but not in an adult chemotherapy population (8). We therefore sought to identify the incidence and risk factors of de novo nephrolithiasis after chemotherapy in adult patients with lymphoproliferative or myeloproliferative disorders.

\section{MATERIALS AND METHODS}

With Institutional Review Board approval, patients with lymphoproliferative or myeloproliferative disorders who received chemotherapy were identified through a retrospective view of the institution's electronic pharmacy and medical records (June 2001 through November 2011) at the University of California San Diego (UCSD) Health System. Searching for specific chemotherapy regimens in the UCSD Siemens and PCSi electronic pharmacy records, we identified patients with hematologic malignancies. The Siemens and PCSi retail pharmacy systems recorded each patient's name, medical record number, start and stop date of chemotherapy, chemotherapy regimen, and location of therapy (outpatient infusion center versus inpatient chemotherapy ward). The UCSD Siemens and PCSi electronic pharmacy database was searched for all chemotherapy regimens for all lymphoproliferative and myeloproliferative disorders treated during this time period (See Appendix 1 for a summary of these chemotherapy regimens).

After including all possible chemotherapy regimens in our query, 2,540 patients were identified. Each medical record was independently reviewed using the Epic medical record system (Verona, Wisconsin) to confirm that each of these patients indeed had a lymphoproliferative or myeloproliferative disorder. After reviewing each medical record and excluding patients who either did not have a hematologic malignancy or who already had a diagnosis of nephrolithiasis, we determined the population at risk to be 1,316 patients.

One hundred percent of patients included underwent CT abdomen/pelvis with IV contrast studies before and after chemotherapy. Patients were followed for up to 10 years with CT abdomen/pelvis with IV contrast studies after their last chemotherapy treatment to calculate incidence. We did not rely on patient's history, but reviewed each CT scan independently and confirmed our review with a radiologist's dictation. Each $\mathrm{CT}$ abdomen/pelvis was independently reviewed by the same physician.

A prior history of nephrolithiasis was determined by a comprehensive chart review of each 
patient's past medical as well as past surgical history. Each note dictated by the patient's oncologist and by their primary care physician was carefully reviewed to assess for prior history of nephrolithiasis. Furthermore, each pretreatment CT study was reviewed to search for history of nephrolithiasis.

Exhaustive manual chart reviews for diagnosis and surgery of nephrolithiasis was performed using Epic to further identify patients with symptomatic stones. All results were confirmed by identifying the stone manually on imaging (abdominal/ pelvic computerized tomography (CT). We excluded any subject with a history of nephrolithiasis prior to chemotherapy initiation to determine the primary outcome, cumulative incidence of de novo kidney stone formation. This was not reported as person-time incidence rate because this measure assumes that the incidence rate is constant over different periods of time.

The secondary outcomes were risk factors for stone formation (expressed as odds ratio with 95\% confidence intervals) derived from demographic and clinical variables. Clinical variables examined included age, race, gender, primary malignancy, diabetes mellitus, hypertension, hyperlipidemia, obesity, allopurinol use, other stone preventing drugs (potassium citrate, thiazide diuretics), peak serum values (uric acid, calcium, potassium, and phosphorous) and trough urinary $\mathrm{pH}$ during chemotherapy. Chemotherapy regimen was not included as a clinical predictor variable because many patients were treated with multiple chemotherapy regimens as a result of relapse, making comparisons difficult. Results of 24-hour urine collections, time to stone formation, size, location, Hounsfield Units (HU) of stone, symptoms of stone presentation, and management of symptomatic stones were also recorded.

Because allopurinol use was more commonly associated with stone formers in our initial analysis, we performed a sub-analysis to determine differences in allopurinol users versus non-allopurinol users.

\section{Statistical analysis}

Comparative statistics were used to compare demographic and disease specific variables for patients who developed de novo stones versus tho- se who did not, and to compare patients who took allopurinol versus those who did not. Independent t-test and Mann-Whitney U test were used for continuous variables depending on distribution, and Chi-square and Fishers exact test were used for categorical variables. Proportion of all predictor variables forming stones was determined with Chi-square testing for significance. Multivariate analysis using binary logistic regression (with backwards log-rank model building) was performed on variables found to be statistically significant on univariate analysis, or of clinical interest to identify predictors of de novo stone formation; only the variables that remained significant on multivariate analysis were included in the final model. All reported p-values were 2-sided, with $\mathrm{p}<0.05$ considered statistically significant. Statistical analyses were performed using SPSS software (version 18.0, SPSS Inc., Chicago IL).

\section{RESULTS}

There were 1,316 patients with either a lymphoproliferative or myeloproliferative disorder and no pre-existing stone disease treated between 2001 and 2011. The incidence of overall and symptomatic de novo nephrolithiasis was 72/1316 (5.5\%) and 24/1316(1.8\%). Lymphoproliferative disorders comprised $68.3 \%$ of the cohort, with the most prevalent disorders being Non-Hodgkin lymphoma and ALL, while acute myeloid leukemia (AML) was the most prevalent myeloproliferative disorder (Figure-1). There were no differences in age, gender, race, rates of obesity, allopurinol utilization, urinary $\mathrm{pH}$, and serum phosphorus levels between stone formers and non-stone formers (Table-1). Proportion of patients with diabetes mellitus $(13.9 \%$ vs. $3 \%, \mathrm{p}<0.001)$, hypertension $(20.8 \%$ vs. $8.8 \%, \mathrm{p}=0.003)$, hyperlipidemia ( $15.3 \%$ vs. $3.7 \%$, $\mathrm{p}<0.001)$, stone prevention drug use $(26.4 \%$ vs. $6.8 \%, \mathrm{p}<0.001$ ), hyperuricemia ( $36.5 \%$ vs. $19.7 \%$, $\mathrm{p}=0.007)$, and hypercalcemia $(26.1 \%$ vs. $12.9 \%$, $\mathrm{p}=0.006$ ) were statistically higher in the stone-formers. Non-Hodgkin lymphoma constituted $28 \%$ of the hematologic malignancies observed in stone formers followed by Hodgkin lymphoma (15\%), diffuse large B cell lymphoma (13\%) and then AML (13\%) (Table-2). There were no differences in 
Figure 1 - Proportion of hematologic malignancies observed in cohort, $n=1316$.

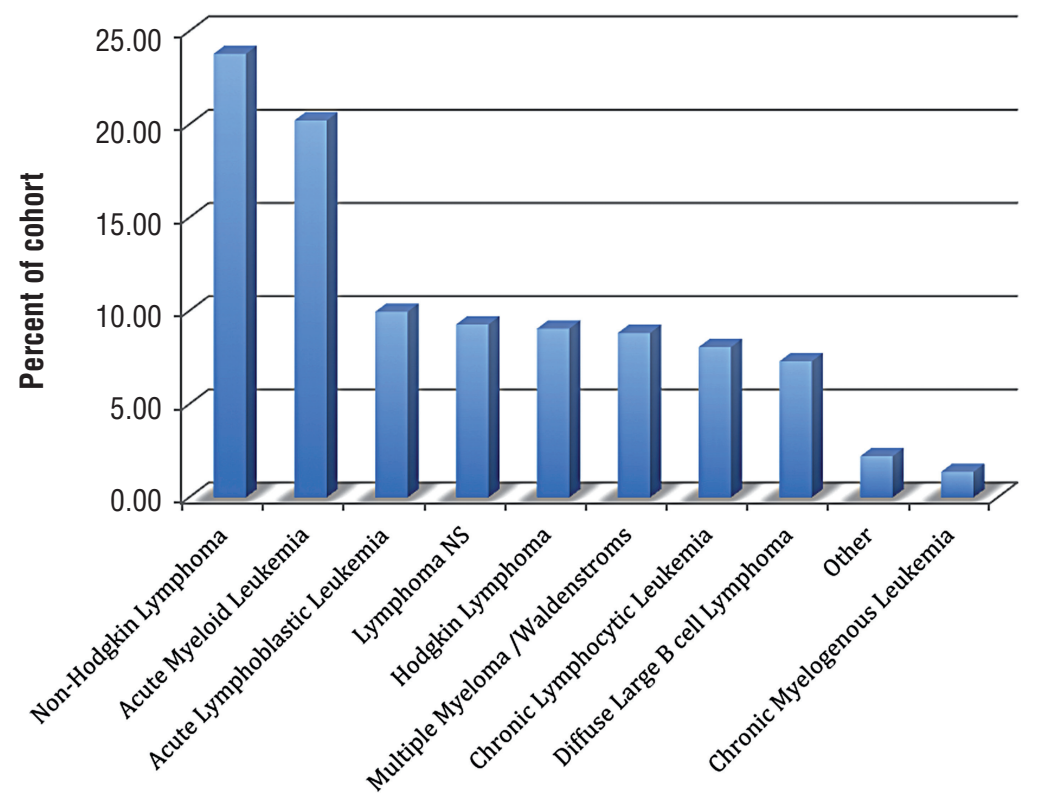

the proportion of primary hematologic malignancies observed in stone formers vs. non-stone formers $(p=0.601)$ (Table-2). Specifically, there were no significant differences in rates of any lymphoproliferative disorder (diffuse large B cell lymphoma, Non-Hodgkins lymphoma, Hodgkins lymphoma, chronic lymphocytic leukemia, acute lymphoblastic leukemia, or multiple myeloma/Waldenstrom's macroglobulinemia) among patients with or without stones, nor were there any significant differences observed in rates of any myeloproliferative disorder (AML or chronic myelogenous leukemia) among patients with or without stones.

Among stone formers, the median urinary $\mathrm{pH}$ was 5.5, the mean serum uric acid was 7.5, calcium was 9.6, potassium was 4.3 , and phosphorus level was $3.8 \mathrm{mg} / \mathrm{dL}$. Median stone size was $3 \mathrm{~mm}$, median $\mathrm{HU}$ was 341, median time from initial chemotherapy to incident stone formation was 3.9 months (1.3-10.7), and 34.8\% of stones were symptomatic.

Only 30 of the 72 stone formers (43.5\%) underwent very elementary 24-hour urine collections, and median 24-hour urinary volume was $1,873 \mathrm{~mL}$. Only 1 of 30 patients undergoing 24hour urine collection had 24-hour urinary uric acid and calcium levels analyzed, and this patient had both hyperuricosuria (urinary uric acid $825 \mathrm{mg}$ ) and hypercalciuria (urinary calcium $334 \mathrm{mg}$ ). No stones were analyzed, however, spot urinalyses performed during chemotherapy demonstrated crystalluria in 11 patients (15.2\%): calcium oxalate crystals observed in 10 (14.3\%) and uric acid crystals observed in $1(1.4 \%)$.

Allopurinol users had different metabolic parameters compared with non-allopurinol users (mean uric acid 7.4 vs. $5.9 \mathrm{mg} / \mathrm{dL}, \mathrm{p}<0.001$, mean potassium 4.3 vs. $4.1 \mathrm{mg} / \mathrm{dL}, \mathrm{p}=0.002$, and mean phosphorus 4.0 vs. $3.7 \mathrm{mg} / \mathrm{dL}, \mathrm{p}=0.018$ respectively). The proportions of diffuse large $B$ cell lymphoma (DLBCL) (11\%vs. 6.6\%, $\mathrm{p}=0.020)$ and CLL $(19.2 \%$ vs. $5.8 \%, \mathrm{p}<0.001)$ were also significantly higher in allopurinol users, while proportions of Hodgkin lymphoma (HL) (5.0\% vs. 9.9\%, $\mathrm{p}=0.020$ ) and acute myeloid leukemia (AML) (14.2\% vs. $21.4 \%, p=0.016)$ were significantly lower. Hyperuricemia (35\% versus $16.1 \%, \mathrm{p}<0.001$, respectively), and hypercalcemia ( $17.6 \%$ vs. $12.8 \%, \mathrm{p}=0.065$ ) were observed more frequently in allopurinol users than non-allopurinol users.

In multivariate analysis, diabetes mellitus $(\mathrm{OR}=6.38, \mathrm{p}<0.001)$, hyperuricemia $(\mathrm{OR}=2.31$, 
Table 1 - Demographics and clinical variables of stone formers and non-stone formers.

\begin{tabular}{|c|c|c|c|}
\hline & \multicolumn{3}{|c|}{ Stones } \\
\hline & No & Yes & $p$-value \\
\hline & $(n=1,244)$ & $(n=72)$ & \\
\hline Mean Age $\pm S D$, years & $51 \pm 15.7$ & $52 \pm 15.2$ & 0.508 \\
\hline Gender & & & 0.454 \\
\hline Male & $765(61.5 \%)$ & $48(66.7 \%)$ & \\
\hline Female & $479(38.5 \%)$ & $24(33.3 \%)$ & \\
\hline Race/Ethnicity & & & 0.459 \\
\hline Caucasian & $515(57.0 \%)$ & $31(63.3 \%)$ & \\
\hline Other & $389(43.0 \%)$ & $18(36.7 \%)$ & \\
\hline Diabetes Mellitus & $37(3.0 \%)$ & $10(13.9 \%)$ & $<0.001^{*}$ \\
\hline HTN & $110(8.8 \%)$ & $15(20.8 \%)$ & $0.003^{*}$ \\
\hline Obesity & $8(0.6 \%)$ & $1(1.4 \%)$ & 0.398 \\
\hline $\mathrm{HL}$ & $46(3.7 \%)$ & $11(15.3 \%)$ & $<0.001^{*}$ \\
\hline Malignancy & & & 0.601 \\
\hline Myeloproliferative & $385(31.2 \%)$ & $20(27.8 \%)$ & \\
\hline Lymphoproliferative & $848(68.8 \%)$ & $52(72.2 \%)$ & \\
\hline Stone Location & & & - \\
\hline Kidney & - & $63(91.3 \%)$ & \\
\hline Ureter & - & $6(8.7 \%)$ & \\
\hline Median Stone Size (IQR), mm & - & $3.0(2.0-5.0)$ & - \\
\hline Median (IQR) Hounsfield Units (HU) of Stone & & $341(254-582)$ & \\
\hline Median time from Initial chemo to stone (IQR), months & - & $3.9(1.3-10.7)$ & - \\
\hline Median time from Immediate prior chemo to stone (IQR), months & - & $1.2(0.4-4.0)$ & - \\
\hline Symptomatic & - & $24(34.8 \%)$ & - \\
\hline Median time from Initial chemo to stone (IQR), months & - & $2.5(0.6-15.4)$ & - \\
\hline Median time from Immediate prior chemo to stone (IQR), months & - & $1.2(0.3-8.7)$ & - \\
\hline Surgery & - & $8(11.6 \%)$ & - \\
\hline Allopurinol & $201(16.2 \%)$ & $18(25.0 \%)$ & 0.071 \\
\hline Stone Preventing Drug (not including Allopurinol) & $84(6.8 \%)$ & $19(26.4 \%)$ & $<0.001^{*}$ \\
\hline 24 hour Urine & - & $30(43.5 \%)$ & - \\
\hline Median 24 hour urine volume (IQR), $\mathrm{mL}$ & - & $1873(1225-3565)$ & - \\
\hline Median Urine $\mathrm{pH}(\mathrm{IQR})$ & $5.5(5.0-6.0)$ & $5.5(5.0-6.0)$ & 0.296 \\
\hline Urine $\mathrm{pH}$ & & & 0.683 \\
\hline$<5.5$ & $339(42.3 \%)$ & $27(45.8 \%)$ & \\
\hline$\geq 5.5$ & $463(57.7 \%)$ & $32(54.2 \%)$ & \\
\hline Mean Serum Uric Acid \pm SD & $6.2 \pm 3.0$ & $7.5 \pm 3.6$ & $0.013^{*}$ \\
\hline Serum Uric Acid (Reference range $3.4-7.0 \mathrm{ng} / \mathrm{dL}$ ) & & & $0.007^{*}$ \\
\hline$<8$ & $519(80.3 \%)$ & $33(63.5 \%)$ & \\
\hline$\geq 8$ & $127(19.7 \%)$ & $19(36.5 \%)$ & \\
\hline Mean Serum Calcium \pm SD & $9.1 \pm 0.9$ & $9.6 \pm 1.5$ & $<0.001^{*}$ \\
\hline Serum Calcium (Reference range $8.6-10.5 \mathrm{ng} / \mathrm{dL}$ ) & & & $0.006^{\star}$ \\
\hline$<10$ & $1051(87.1 \%)$ & $51(73.9 \%)$ & \\
\hline$\geq 10$ & $156(12.9 \%)$ & $18(26.1 \%)$ & \\
\hline Mean Serum Potassium \pm SD (Reference range 3.5-5.1 mmol/L) & $4.1 \pm 0.6$ & $4.3 \pm 0.6$ & $0.039 *$ \\
\hline Mean Serum Phosphorous \pm SD (Reference range $2.7-4.5$ ng/dL) & $3.8 \pm 1.2$ & $3.8 \pm 1.0$ & 0.771 \\
\hline Deceased & $146(11.7 \%)$ & $9(12.5 \%)$ & 0.850 \\
\hline
\end{tabular}


Table 2 - Rates of primary malignancy in stone formers vs. non-stone formers.

\begin{tabular}{lccc}
\hline & \multicolumn{2}{l}{ Stones } \\
\hline & No & Yes & p-value \\
\hline Lymphoproliferative Disorders & $(\mathrm{n}=1,244)$ & $(\mathrm{n}=72)$ & \\
\hline Diffuse Large B cell Lymphoma (DLBL) & $947(76.1 \%)$ & $56(77.8 \%)$ & 0.601 \\
Non-Hodgkin Lymphoma (NHL) & $87(7 \%)$ & $9(12.5 \%)$ & 0.098 \\
Hodgkin Lymphoma (HL) & $293(23.6 \%)$ & $20(27.8 \%)$ & 0.396 \\
Lymphoma NS & $108(8.7 \%)$ & $11(15.3 \%)$ & 0.086 \\
Chronic Lymphocytic Leukemia (CLL) & $120(9.6 \%)$ & $2(2.8 \%)$ & 0.057 \\
Acute Lymphoblastic Leukemia (ALL) & $100(8 \%)$ & $6(8.3 \%)$ & 0.826 \\
Multiple Myeloma (MM)/Waldenstroms & $127(10.2 \%)$ & $4(5.6 \%)$ & 0.308 \\
Myeloproliferative Disorders & $112(9 \%)$ & $4(5.6 \%)$ & 0.397 \\
Acute Myeloid Leukemia (AML) & $274(22 \%)$ & $10(13.9 \%)$ & 0.601 \\
Chronic Myelogenous Leukemia (CML) & $257(20.7 \%)$ & $9(12.5 \%)$ & 0.099 \\
Other & $17(1.4 \%)$ & $1(1.4 \%)$ & 1.000 \\
\hline
\end{tabular}

Other: T-cell prolymphocytic leukemia, Aplastic anemia, chronic congenital neutropenia, CVID

HIV with Autoimmune hemolytic anemia, idiopathic hypogammaglobulinemia, leukemia, Myelodysplastic syndrome, Myelofibrosis, myeloid sarcoma, Pancytopenia, r/o MDS, plasma cell leukemia, Polycythemia Vera, t-cell leukemia

$\mathrm{p}=0.007)$, and hypercalcemia $(\mathrm{OR}=2.14, \mathrm{p}=0.022)$ at time of chemotherapy predicted de novo stone.

\section{DISCUSSION}

The findings from this study spanning 10 years support the hypothesis that metabolic derangements during chemotherapy are associated with an increased risk of nephrolithiasis. Previous extensive reports demonstrating this include a pediatric study of over 2,000 children treated for ALL and a Korean study of over 900 adults who were treated for both lymphoproliferative and myeloproliferative disorders $(5,8)$. Both investigations demonstrated that the incidence of nephrolithiasis in these populations was significantly higher than in the general population. Until these reports, the claim of increased nephrolithiasis risk with hematologic malignancy had been substantiated by only case reports and the plausible pathophysiologic theory of endogenous nucleotide catabolism.

The investigators of the pediatric stone study postulated that stone formation was associated with chemotherapy, but perhaps more importantly was due to glucocorticoid therapy. Steroids are used in multiple contexts for lymphoproliferative diseases and can increase the risk of nephrolithiasis by decreasing renal calcium absorption. The authors cited the predominance of calcium-based stones as opposed to uric acid stones as evidence supporting the steroid-nephrolithiasis hypothesis (8). However, almost half of stone analyses in the Korean adult study showed uric acid stones despite the common use of glucocorticoid therapy (5).

Our study included a predominance of calcium oxalate over uric acid crystals, although no stones were formally analyzed, and the median $\mathrm{HU}$ of de novo stones was 341, suggesting a mixture of 
both calcium and uric acid stone compositions (9). These findings are consistent with our analysis of nephrolithiasis risk factors, which demonstrated in multivariate analysis that diabetes mellitus, hyperuricemia, or hypercalcemia significantly increased risk of nephrolithiasis. The presence of hypercalcemia and hyperuricemia permits heterogeneous nucleation and subsequent calcium stone formation (10). Diabetes is thought to be a risk factor for uric acid calculi due to insulin resistance leading to low urinary $\mathrm{pH}$ associated with defective ammonia synthesis occurring in the proximal tubule cell as well as ammonium transport into the renal tubular lumen (11). The role of diabetes mellitus in our study is significant, as it was associated with an over 6-fold increased risk of nephrolithiasis. Allopurinol is a xanthine oxidase inhibitor frequently used prophylactically to decrease the uric acid production prior to instituting chemotherapy for patients at risk for TLS (12). In a double blinded randomized prospective trial, allopurinol utilization decreased the number of stone events while increasing the time to recurrence of stone event among calcium oxalate stone formers with hyperuricosuria on 24-hour urine collections, substantiating its preventive role in patients at risk for calcium oxalate nephrolithiasis (13). Published guidelines regarding the prevention and management of TLS include specific recommendations for the appropriate utilization of allopurinol (14). Risk factors for TLS include tumor type (Burkitt's lymphoma, lymphoblastic lymphoma, diffuse large cell lymphoma, ALL), tumor burden/ extent of disease defined by elevated WBC $>25,000$ and/or bulky nodal disease $>10 \mathrm{~cm}$, preexisting renal failure, and baseline uric acid $\geq 7.5 \mathrm{mg} / \mathrm{dL}$ (14).

These high-risk patients are recommended to undergo aggressive hydration, urinary alkalinization, diuresis, and allopurinol or recombinant urate oxidase (rasburicase) prophylaxis. Our data demonstrated patients at higher risk for TLS received allopurinol more frequently than those at lower risk, as one would expect, but in multivariate analysis use of allopurinol did not decrease incident stone risk, presumably due to selection bias. Given this and our finding that hyperuricemia is a risk factor for stone formation in this population, recombinant urate oxidase (rasburicase) may offer an advantage, as it catalyzes the conversion of uric acid to allantoin, which is $5-10$ times more soluble in urine (14). Rasburicase has been well studied in both pediatric and adult patient populations at risk for TLS, demonstrating a significantly more rapid lowering of serum uric acid levels compared to allopurinol (15-17). Current guidelines for management of TLS recommend rasburicase in pediatric patients at high risk for TLS and in adults with hyperuricemia diagnosed with TLS or refractory to allopurinol (14). Our findings suggest that prompt and effective treatment of hyperuricemia may prevent upper tract stone formation and reduce the incidence of nephrolithiasis.

Unfortunately, our study highlights both the low number of 24-hour urine collections $(43.5 \%)$ as well as the lack of necessary detail in these 24-hour urine collections (3.33\%) performed in high-risk stone formers. Urological consultations were infrequently obtained in this patient population, and as such, metabolic stone evaluations were rarely performed in these patients. While it would have been incredibly useful to have a 24-hour urine collection in every single stone former, the absence of this data provides an area of opportunity for urologists to improve the care of these patients. Urologists should be consulted in the care of such patients to appropriately work up the etiology of stone disease, and thereby prevent future stones from occurring.

Inherent limitations of this retrospective study are acknowledged, specifically, selection bias was observed when comparing patients receiving allopurinol prophylaxis. Patients in our study attended regular office visits and underwent frequent cross-sectional imaging for oncologic management and surveillance, resulting in more opportunities to diagnose asymptomatic nephrolithiasis. Moreover, patients may have been more likely to report symptoms of abdominal or flank pain at follow-up visits, increasing the likelihood of diagnosis of nephrolithiasis.

\section{CONCLUSIONS}

We report the incidence of de novo nephrolithiasis in patients with lymphoproliferative or myeloproliferative disorders undergoing chemotherapy. This study also identifies diabetes 
mellitus, as well as hyperuricemia and hypercalcemia at time of chemotherapy as risk factors for nephrolithiasis that should assist oncologists in appropriately selecting patients for prophylaxis, while also providing urologists an earlier opportunity to collaborate and assist in treatment and evaluation of nephrolithiasis.

\section{ABBREVIATIONS}

ALL = Acute Lymphoblastic Leukemia

$\mathrm{AML}=$ Acute Myeloid Leukemia

CLL = Chronic Lymphocytic Leukemia

DLBL = Diffuse Large B cell Lymphoma

HL = Hodgkins lymphoma

$\mathrm{HU}=$ Hounsfield Unit

$\mathrm{MM}=$ Multiple Myeloma

NHL $=$ Non Hodgkins Lymphoma

TLS $=$ Tumor lysis syndrome

\section{CONFLICT OF INTEREST}

None declared.

\section{REFERENCES}

1. Cairo MS, Coiffier B, Reiter A, Younes A; TLS Expert Panel: Recommendations for the evaluation of risk and prophylaxis of tumour lysis syndrome (TLS) in adults and children with malignant diseases: an expert TLS panel consensus. $\mathrm{Br} \mathrm{J}$ Haematol. 2010;149:578-86.

2. Abu-Alfa AK, Younes A: Tumor lysis syndrome and acute kidney injury: evaluation, prevention, and management. Am J Kidney Dis. 2010;55(5 Suppl3):S1-13.

3. Howard SC, Ribiero RC, Pui C-H. Acute complications in childhood leukemias. cambridge, UK: Cambridge University Press, 2006.

4. Shimada M, Johnson RJ, May WS Jr, Lingegowda V, Sood P, Nakagawa T, et al.: A novel role for uric acid in acute kidney injury associated with tumour lysis syndrome. Nephrol Dial Transplant. 2009;24:2960-4.

5. Kim SW, Kim SD, Yoo JM, Cho YH, Sohn DW: Urolithiasis in patients suffering from malignant hematologic diseases. Yonsei Med J. 2010;51:244-7.

6. Shiozawa Y, Sakaguchi S, Sakakibara 0, Yagishita K, Saito M, Yamashiro Y: Urolithiasis in an acute lymphoblastic leukemia child during induction chemotherapy. Pediatr Hematol Oncol. 2008;25:359-63.
7. Jaing TH, Hung IJ, Lin CJ, Chiu CH, Luo CC, Wang CJ: Acute myeloid leukemia complicated with staghorn calculus. Jpn J Clin Oncol. 2002;32:365-7.

8. Howard SC, Kaplan SD, Razzouk BI, Rivera GK, Sandlund JT, Ribeiro RC: Urolithiasis in pediatric patients with acute lymphoblastic leukemia. Leukemia. 2003;17:541-6.

9. Nakada SY, Hoff DG, Attai S, Heisey D, Blankenbaker $D$, Pozniak M: Determination of stone composition by noncontrast spiral computed tomography in the clinical setting. Urology. 2000;55:816-9.

10. Zerwekh JE, Hwang TI, Poindexter J, Hill K, Wendell G, Pak CY: Modulation by calcium of the inhibitor activity of naturally occurring urinary inhibitors. Kidney Int. 1988;33:1005-8.

11. Maalouf NM, Sakhaee K, Parks JH, Coe FL, Adams-Huet $\mathrm{B}$, Pak CY: Association of urinary $\mathrm{pH}$ with body weight in nephrolithiasis. Kidney Int. 2004; 65: 1422-5.

12. Hochberg J, Cairon MS: Tumor lysis syndrome: current perspective. Haematologica. 2008; 93: 9-13.

13. Ettinger B, Tang A, Citron JT, Livermore B, Williams T: Randomized trial of allopurinol in the prevention of calcium oxalate calculi. N Engl J Med. 1986;315:1386-9.

14. Coiffier B, Altman A, Pui CH, Younes A, Cairo MS: Guidelines for the management of pediatric and adult tumor lysis syndrome: an evidence-based review. J Clin Oncol. 2008 Jun 1;26(16):2767-78. Erratum in: J Clin Oncol. 2010;28:708.

15. Pui $\mathrm{CH}$, Mahmoud HH, Wiley JM, Woods GM, Leverger $G$, Camitta B, et al:: Recombinant urate oxidase for the prophylaxis or treatment of hyperuricemia in patients With leukemia or Iymphoma. J Clin Oncol. 2001;19:697-704.

16. Goldman SC, Holcenberg JS, Finklestein JZ, Hutchinson R, Kreissman S, Johnson FL, et al.: A randomized comparison between rasburicase and allopurinol in children with lymphoma or leukemia at high risk for tumor lysis. Blood. 2001;97:2998-3003.

17. Coiffier B, Mounier N, Bologna S, Fermé C, Tilly $H$, Sonet $A$, et al.: Efficacy and safety of rasburicase (recombinant urate oxidase) for the prevention and treatment of hyperuricemia during induction chemotherapy of aggressive non-Hodgkin's lymphoma: results of the GRAAL1 (Groupe d'Etude des Lymphomes de l'Adulte Trial on Rasburicase Activity in Adult Lymphoma) study. J Clin Oncol. 2003;21:4402-6.

Correspondence address: Roger L. Sur, MD

UC San Diego Comprehensive Kidney Stone Center Department of Urology 200 W Arbor Dr. \#8897

San Diego, CA 92103-8897 USA

Fax: +1 619 543-6573

E-mail: rlsur@ucsd.edu 


\section{APPENDIX 1: LIST OF CHEMOTHERAPY REGIMENS}

\section{Lymphoma}

1. Cyclophosphamide, doxorubicin, vincristine and prednisone (CHOP) with/without rituximab

2. Etoposide, prednisone, vincristine, doxorubicin, and cyclophosphamide (EPOCH) with/without rituximab

3. Ifosfamide, carboplatin, and etoposide (ICE) with or without rituximab

4. Doxorubicin, bleomycin, vinblastine, and dacarbazine (ABVD)

5. Nitrogen mustard, doxorubicin, vinblastine, vincristine, bleomycin, etoposide, and prednisone (Stanford V)

Acute myelogenous leukemia

1. Cytarabine and idarubicin or daunorubicin $(7+3)$

2. Fludarabine, cytarabine, idarubicin, and filgrastim (FLAG)

3. All trans-retinoic acid (ATRA)

4. Chronic myelogenous leukemia

5. Imatinib, dasatinib and nilotinib 